\title{
Simulated microgravity hampers Notch signaling in the fight against myocardial ischemia-reperfusion injury
}

\author{
SHUAI JIANG ${ }^{*}$, XING-CHENG ZHAO* ${ }^{*}$ BO JIAO, ZHI-JIE YUE and ZHI-BIN YU \\ Department of Aerospace Physiology, Fourth Military Medical University, Xi'an, Shaanxi 710032, P.R. China
}

Received April 25, 2017; Accepted October 23, 2017

DOI: $10.3892 / \mathrm{mmr} .2018 .8489$

\begin{abstract}
The gravitational field is an important determinant of cardiovascular function. Exposure to microgravity during spaceflight may lead to a series of maladaptive alterations in the cardiovascular system. The authors have previously demonstrated that microgravity can increase the susceptibility to myocardial ischemia-reperfusion (IR) injury under simulated microgravity. Although Notch1 signaling protects against myocardial IR injury, whether Notch1 protects against myocardial IR injury under simulated weightlessness remains unknown. The present study is designed to investigate the role of the Notch1 receptor in myocardial IR injury under simulated weightlessness. The differences in Notch signaling expression and myocardial infarct size following myocardial IR were compared between normal rats and tail-suspended rats that were kept in $30^{\circ}$ head-down tilt and hindlimb unloading position. The data revealed low expression levels of Notch1 receptor and its endogenous ligand Jagged1 in normal adult rat hearts. However, significantly higher expression of Notch1 was observed in the border zone compared with the infarcted area and the remote zone following myocardial
\end{abstract}

Correspondence to: Professor Zhi-Bin Yu, Department of Aerospace Physiology, Fourth Military Medical University, 169 Changlexi Road, Xi'an, Shaanxi 710032, P.R. China

E-mail: yuzbfmmu@126.com

*Contributed equally

Abbreviations: BSA, bovine serum albumin; CHD, coronary heart disease; CON, control; CON-IR-3h, 3-h reperfusion without suspension; CON-IR-7d, 7-day reperfusion without suspension; Cx43, connexin 43; IR, ischemia-reperfusion; LAD, left anterior descending coronary artery; NF- $\kappa \mathrm{B}$, nuclear transcription factor- $\kappa \mathrm{B}$; NICD, Notch intracellular domain; PBS, phosphate buffered saline; PVDF, polyvinylidene fluoride; SD, Sprague-Dawley; SEM, standard error of the mean; SUS, tail-suspended; SUS-IR-3h, 3-h reperfusion following 4-week suspension; SUS-IR-7d, 7-day reperfusion following 4-week suspension; TBS, Tris-buffered saline; TTC, triphenyltetrazolium chloride; WGA, wheat germ agglutinin

Key words: simulated microgravity, myocardial infarction, ischemia-reperfusion injury, border zone, Notch signaling
IR. Notch1 expression was notably reduced in the infarcted hearts of tail-suspended rats compared with the control group. Conversely, the myocardial infarct size was significantly increased in tail-suspended rats compared with the control rats. In conclusion, these data suggested that the proper function of Notch signaling may be hampered under simulated microgravity.

\section{Introduction}

Cardiovascular disease is the leading non-communicable disease in terms of mortality; in 2012 there were 17.5 million moralities caused by cardiovascular diseases, among which $\sim 7.4$ million were caused by coronary heart disease (CHD) (1). The detrimental effects of acute myocardial ischemia-reperfusion (IR) injury are usually responsible for the outcome of CHD (2). During spaceflight, exposure to microgravity may lead to a series of maladaptive alterations in a number of organs, resulting from the redistribution of body fluids towards the head, which is caused by the considerable stress on the cardiovascular system (3). Data from our previous study revealed that microgravity increases susceptibility to myocardial IR injury in tail-suspended rats (4); however, the underlying mechanisms have not been fully elucidated.

The Notch gene was originally cloned from Drosophila melanogaster $>30$ years ago; it encodes a family of highly conserved transmembrane surface receptors (5). Mammals express 4 Notch receptors, Notch1-Notch4, and 5 Notch ligands, including Delta-like (DLL)1, DLL3, DLL4, Jagged (JAG)1 and JAG2. The binding of a ligand to a receptor triggers the proteolytic cleavage and release of the Notch intracellular domain (NICD), which is the active form of Notch (6). Following translocation into the nucleus, NICD functions to regulate the transcription of its downstream target genes, including HES family basic helix-loop-helix transcription factor (HES) and HES-related with YRPW motif (HEY), which in turn modulate cell proliferation, apoptosis and the expression of transcription factors of the nuclear transcription factor- $\kappa \mathrm{B}(\mathrm{NF}-\kappa \mathrm{B})$ family (7). The Notch signaling pathway has been revealed to participate in various cellular behaviors and processes, including cell proliferation, apoptosis, differentiation and adhesion by regulating communication between adjacent cells (8), thus indicating its important role in controlling the development of organs and tissues. 
Notably, a previous study demonstrated that a deficiency in Notch signaling aggravates hepatic IR injury, whereas induced Notch activation decreases cell apoptosis following hepatic IR (9), which suggested that Notch may serve a crucial role in adult animals. Among the Notch receptors and ligands, Notch1 and JAG1 are the predominant forms expressed in adult heart (10). Numerous previous studies have reported that the Notch1 signal exhibits significant enhancement following myocardial IR, suggesting that cardiac Notch1 signaling may be endogenously activated and may exert its beneficial effects during myocardial IR (11-13). Nevertheless, whether Notch1 protects against myocardial IR injury under simulated weightlessness remains unclear and requires further investigation.

Therefore, the present study was designed to investigate the role of the Notch1 receptor in myocardial IR under simulated weightlessness. The temporal and spatial characteristics of Notch1 expression in myocardial IR were determined by detecting the extracellular domain of Notch1, where the signaling pathway was initiated. Furthermore, it was demonstrated that simulated weightlessness aggravated myocardial IR injury by downregulating Notch. These data may add to the understanding of the mechanisms of myocardial IR injury in a weightless environment, which may aid in the design of therapies for astronauts or other members who work on the space station.

\section{Materials and methods}

Chemicals and reagents. The extracellular domain of Notch receptors consists of 29-36 multiple epidermal growth factor-like motifs that are responsible for ligand interaction and 3 LIN-12/Notch motifs that are responsible for precluding receptor activation without receptor-ligand engagement $(14,15)$. The NICD is the activated form of Notch1 that directly regulates the transcription of downstream genes (7). Therefore, antibodies used against the extracellular domain of Notch1 may indicate how and where Notch1 is distributed. Primary antibodies against Notch 1 extracellular domain (cat. no. sc-23299) and JAG1 (cat. no. sc-8303) and connexin 43 (Cx43; cat. no. sc-13558) were purchased from Santa Cruz Biotechnology, Inc. (Dallas, TX, USA), and anti- $\beta$-actin (cat. no. A5441) was purchased from Sigma-Aldrich (Merck KGaA, Darmstadt, Germany). The membrane marker Texas Red $^{\circledR}$-X-conjugated wheat germ agglutinin (WGA; cat. no. W21405) was purchased from Molecular Probes (Thermo Fisher Scientific, Inc., Waltham, MA, USA). Cx43 and WGA were used to distinguish between the different zones of infarcted myocardium under microscopy. Unless otherwise indicated, all chemicals were purchased from Sigma-Aldrich (Merck KGaA).

Animals and samples. All Sprague-Dawley (SD) rats $(n=44)$ were obtained from Beijing Vital River Laboratory Animal Technology Co., Ltd. (Beijing, China). All the animals were fed and provided with water ad libitum in an environment with temperature of $22 \pm 1^{\circ} \mathrm{C}$, relative humidity of $50 \pm 1 \%$ and a $12-\mathrm{h}$ light/dark cycle. All animal studies, including the rat euthanasia by carbon dioxide asphyxiation $\left(90 \% \mathrm{CO}_{2}, 5 \mathrm{~min}\right)$, were performed in compliance with the regulations and guidelines, and approved by the Ethics Committee of The Fourth Military Medical University institutional animal care and were conducted according to the AAALAC (https://www.aaalac.org/) and the IACUC (https://www.iacuc.org/) guidelines.

The antibody against Notch1 in the present study has rarely been used before to detect myocardial Notch1. Therefore, the feasibility of using this antibody to detect myocardial Notch1 expression had to be validated. As the liver is one of the organs where Notch is abundantly expressed in the adult stage, hepatic Notch1 expression was used as the positive control for the validation of the experimental method, as well as for further comparisons of Notch expression between other groups. Accordingly, hearts and livers were sourced from male SD rats that were 14 days embryonic (embryonic), 3 days old (neonatal) and 8 weeks old (adult) ( $n=4$ rats/age group) to reveal the expression patterns of the Notch signal. Additionally, 32 adult rats were used to elucidate the effect of simulated microgravity on the expression of Notch signal after myocardial IR.

Tail-suspension model and IR model in vivo. Adult rats (weight, 200-250 g) were randomly divided into the following 6 groups prior to tail-suspension and surgery: i) Control 3-h IR without suspension (CON-IR-3h, $\mathrm{n}=4)$; ii) Control 7-day IR without suspension (CON-IR-7d, $\mathrm{n}=8$ ); iii) 3-h IR following 4-week suspension (SUS-IR-3h, $n=4$ ); iv) 7-day IR following 4-week suspension (SUS-IR-7d, n=8); v) sham without suspension (CON-Sham, $n=4)$; vi) and sham following 4-week suspension (SUS-Sham, n=4) (16). Tail-suspended rats were maintained in a $30^{\circ}$ head-down tilt and hindlimb unloading position for 4 weeks (17). Rats were anesthetized with sodium pentobarbital (40 mg/kg, intraperitoneal; Sigma-Aldrich; Merck KGaA) prior to IR surgery. Myocardial ischemia was induced by ligation of the left anterior descending coronary artery (LAD) with a slipknot, which was subsequently removed 30 min later (18). Sham-operated control rats underwent the same surgical procedures except that the suture placed under the LAD was not tied. SUS-IR-7d rats were returned to the tail-suspension state post-surgery.

Immunofluorescent histochemistry and confocal analysis. Myocardial and hepatic samples sourced from rats were cut into $1 \mathrm{~mm}^{3}$ pieces and frozen with optimum cutting temperature compound and isopentane in liquid nitrogen (Sigma-Aldrich; Merck kGaA). Tissue sections $(10 \mu \mathrm{m})$ were obtained in a freezing cryostat at $-20^{\circ} \mathrm{C}$. Sections were air dried at room temperature, fixed in ice-cold acetone for $30 \mathrm{~min}$, permeabilized in the phosphate buffered saline [PBS; $135 \mathrm{mM} \mathrm{NaCl}, 10 \mathrm{mM}$ sodium phosphate, $(\mathrm{pH} 7.0)]$ including $0.1 \%$ Triton $\mathrm{X}-100$ at room temperature for $30 \mathrm{~min}$, and subsequently blocked in $1 \%$ bovine serum albumin (BSA; cat. no. 9048-46-8; Ameresco, Inc., Framingham, MA, USA) in PBS at room temperature for $60 \mathrm{~min}$. Sections were incubated with anti-Notch1 (1:50), anti-JAG1 (1:50) or anti-Cx43 (1:50) primary antibodies at $4^{\circ} \mathrm{C}$ overnight. Slides were rinsed twice in PBS and incubated with rabbit anti-goat Alexa Fluor 488-conjugated immunoglobulin IgG (1:800; cat. no. A27012; Invitrogen; Thermo Fisher Scientific, Inc.), goat anti-rabbit Alexa Fluor 488-conjugated immunoglobulin IgG (1:800; 
cat. no. A27034; Invitrogen; Thermo Fisher Scientific, Inc.), and goat anti-mouse Cy3-conjugated IgG (1:1,000; cat. no. A0521; Beyotime Institute of Biotechnology) at $37^{\circ} \mathrm{C}$ for $60 \mathrm{~min}$. Tissue sections were counterstained with Hoechst $33258(1 \mu \mathrm{g} / \mathrm{ml})$ and WGA $(5 \mu \mathrm{g} / \mathrm{ml})$ at $37^{\circ} \mathrm{C}$ for $8 \mathrm{~min}$. Stained sections were observed using an Olympus FV1000 laser-scanning confocal microscope (Olympus Corporation, Tokyo, Japan) equipped with the FV10-ASW 3.1. Images were captured using a x60 magnification water objective. Optical densitometric analysis of target protein expression was performed using the FV10-ASW 3.1 (Olympus Corporation, Tokyo, Japan). For each section, mean fluorescence was calculated from three separate fields per heart.

Protein extraction and western blot analysis. The heart samples ( $\mathrm{n}=4$ rats) were collected from the CON-IR-7d group 7 days following myocardial IR surgery to confirm and limit assessment to the spatial features of the border zone. The infarcted hearts were then sectioned horizontally into 6 pieces from apex to base, among which the second section was usually the most typical for the border zone division (Fig. 1A). According to previous studies $(19,20)$, the ischemic, but still viable, 'border zone' was $\sim 2 \mathrm{~mm}$ away from the border of the macroscopic (pink or white colored) infarct zone (Fig. 1B). Thus, the myocardial protein extracts from border, remote, and infarct zone of the second section were resolved by SDS-PAGE using 12\% Laemmli gels, as described previously (21). Following electrophoresis, proteins were electrically transferred to polyvinylidene fluoride membrane using a Bio-Rad semi-dry transfer apparatus (Bio-Rad Laboratories, Inc., Hercules, CA, USA). The membranes were blocked with $1 \%$ BSA in Tris-buffered saline [TBS; $150 \mathrm{mM} \mathrm{NaCl}, 50 \mathrm{mM}$ Tris- $\mathrm{HCl},(\mathrm{pH}$ 7.5)] and were incubated with a goat polyclonal antibody against the extracellular domain of Notch1 $(1: 1,000)$ and mouse monoclonal anti- $\beta$-actin $(1: 4,000)$ in TBS containing $0.1 \%$ BSA at $4^{\circ} \mathrm{C}$ overnight. The membranes were incubated with IRDye $800 \mathrm{CW}$-conjugated donkey-anti goat (1:10,000; cat. no. 926-32214; LI-COR, Inc.) or IRDye $800 \mathrm{CW}$-conjugated goat-anti mouse secondary antibodies (1:10,000; cat. no. 926-32210; LI-COR, Inc.) for $90 \mathrm{~min}$ at room temperature, and were visualized using an Odyssey scanner (LI-COR Biosciences, Lincoln, NE, USA). Quantification analysis of blots was performed with the ImageJ software v1.43u (National Institutes of Health, Bethesda, MD, USA). Notch1 expression was normalized to $\beta$-actin.

Myocardial infarct size. Hearts were arrested in diastole by perfusing with cardioplegic solution $(25 \mathrm{mmol} / \mathrm{l} \mathrm{KCl}$, 5\% dextrose in PBS) at the end of reperfusion. To distinguish between viable and infarcted tissue, hearts were perfused and incubated in $1 \%$ triphenyltetrazolium chloride Sigma-Aldrich (Merck KGaA; TTC, in PBS, $(\mathrm{pH} 7.4)$ ] for $10 \mathrm{~min}$ at $37^{\circ} \mathrm{C}(22)$. The base and the right ventricular free wall were dissected, and the left ventricle was frozen at $-20^{\circ} \mathrm{C}$ for $2 \mathrm{~h}$ and the heart was subsequently sectioned from the top to the bottom into coronal sections. The sections were fixed at $4^{\circ} \mathrm{C}$ overnight in $4 \%$ paraformaldehyde; the sections were weighed to calculate the total mass of the left ventricle and the sides that touched the bottom of the container were imaged the next day. Following incubation with TTC, viable tissue was stained red, whereas
A
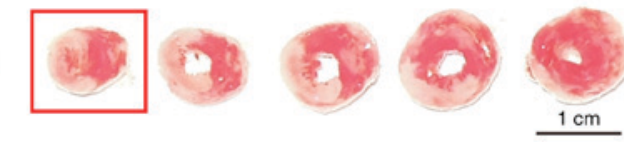

B

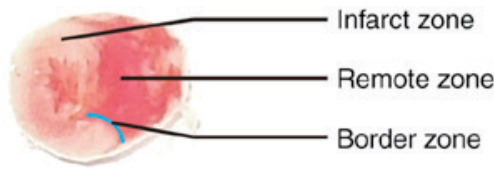

Figure 1. Remote, border and infarct zones in left ventricular slices from a SUS-IR-3h group triphenyltetrazolium chloride stained heart. (A) The infarcted hearts were sectioned horizontally into 6 pieces from apex to base. (B) The remote zone is pink and the infarcted zone is white. The border zone sample was excised from $\sim 2 \mathrm{~mm}$ near the infarct zone in the second coronal section of the left ventricle.

necrotic (infarcted) tissue turned white. The total mass of the left ventricle was measured by adding the masses of the individual slices. The infarct size was then expressed as the percentage of the mass of the left ventricle. The areas of infarct were analyzed using Image J software v1.43u.

Statistical analysis. Data were statistically analyzed with the SPSS 17.0 software (SPSS Inc., Chicago, IL, USA). Data are presented as the mean \pm standard error of the mean from at least three independent experiments. Student's t-test was used for paired observations. Comparisons were performed using two-way ANOVA for experiments with more than two subgroups. $\mathrm{P}<0.05$ was considered to indicate a statistically significant difference.

\section{Results}

Hepatic Notch expression gradually decreases during development. Notch1 protein was abundantly expressed in embryonic liver, but it was notably decreased in neonatal and adult liver tissues (Fig. 2). In the adult liver, Notch1 expression was observed in a ring-shaped manner around each hepatocyte, which indicated that the protein was localized to the cell membrane. This concurs with the use of the Notch antibody in the present study against the extracellular domain, which may better indicate how and where Notch 1 signal gets distributed and explains why Notch1 signal in the present study always lies near the cell membrane. Similarly, JAG1 expression was also intensely expressed in the embryonic liver (Fig. 3), whereas the levels of hepatic JAG1 expression were decreased in neonatal livers and became even lower at the adult stage. In the adult liver, JAG1 expression was distributed in a similar ring-shaped pattern to Notch1 expression (Fig. 2). In conclusion, these results revealed that Notch expression in rat liver gradually decreased during development.

Notch is expressed at lower levels in heart compared with liver. It must be noted that myocardial Notch1 expression has rarely been studied using the Notch1 antibody against the extracellular domain. In addition, the expression levels of Notch signal in the adult and neonatal hearts are too low to be visually defined. Therefore, the expression of the Notch signal in both the liver and heart at the three different developmental periods (Figs. 2-5) has been compared to validate 

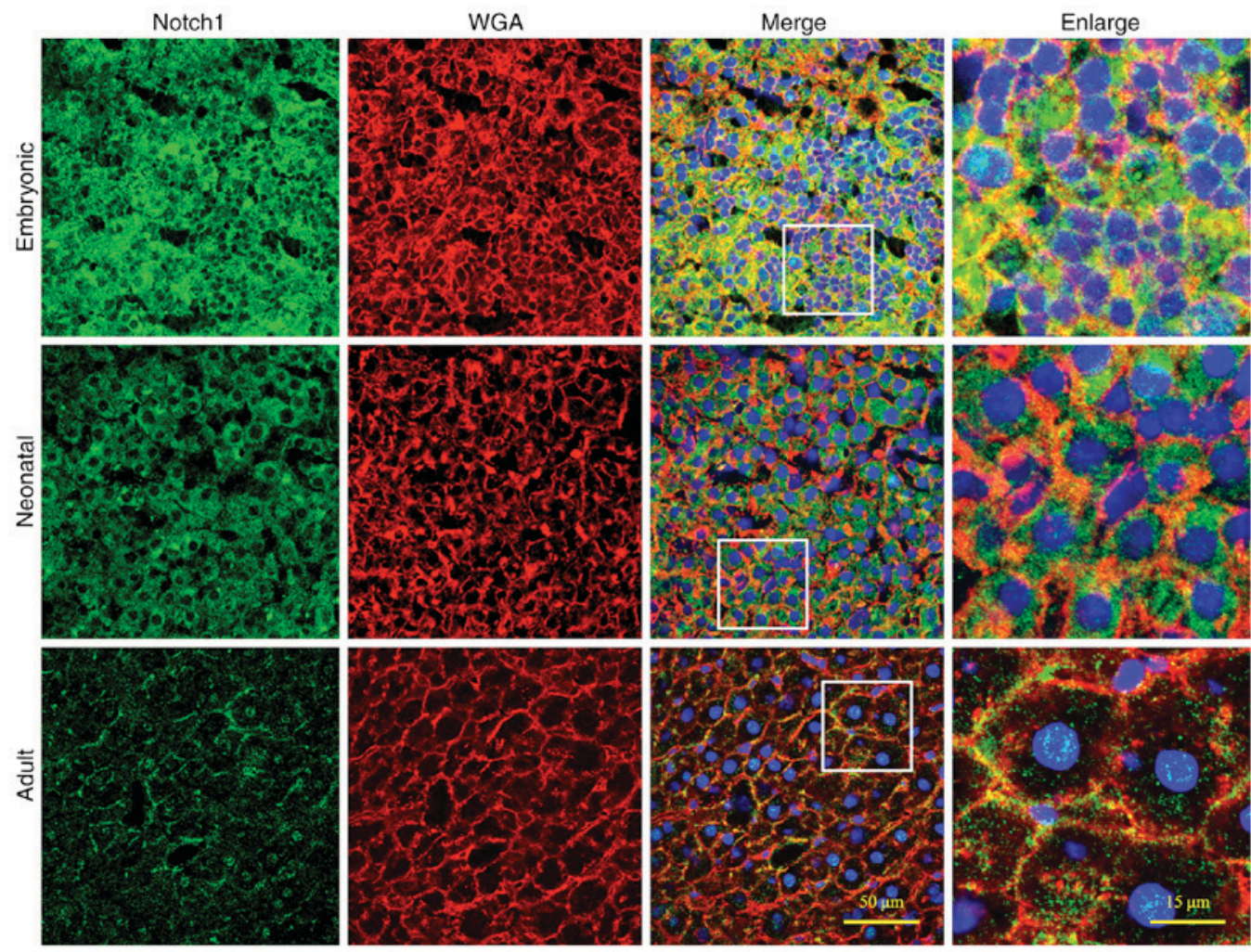

Figure 2. Expression of hepatic Notch1 at different stages of rat development. Frozen sections (magnification, x60) of liver from embryonic, neonatal and adult rats were stained for the extracellular domain of Notch1 (green), membrane (WGA, red) and nucleus (Hoechst, blue). The size of hepatocytes increased over developmental time, and the intensity of Notch1 expression decreased from the embryonic to the adult stage. The enlarged area (magnification, $\mathrm{x} 200$ ) demonstrates the ring-shaped distribution of Notch1 around hepatocytes, which was particularly enhanced in the embryonic and neonatal stages. WGA, wheat germ agglutinin.

the feasibility of using these antibodies to detect myocardial Notch signal, with the expression level in the adult liver being used as a baseline. Lower expression of Notch1 was observed in the hearts of the neonatal and adult rats compared with expression in the liver at these respective stages (Fig. 4A). Even at the embryonic stage of the rat heart, Notch1 expression level was significantly lower compared with the adult liver. Following statistical comparison, it was demonstrated that the expression levels of Notch1 in liver were $>10$ times higher compared with the heart at the same developmental stage. Notably, Notch1 expression levels of both neonatal and adult hearts were significantly less than that of embryonic hearts ( $\mathrm{P}<0.01$; Fig. 4B). Similarly, the levels of JAG1 protein expression were low in the embryonic heart but significantly higher than in neonatal or adult hearts, in both of which JAG1 was almost undetectable ( $\mathrm{P}<0.01$; Fig. $5 \mathrm{~A})$. Furthermore, at the same stage, cardiac JAG1 expression levels were significantly lower compared with the hepatic JAG1 expression levels (P<0.01; Fig. 5B).

Notchl expression is enhanced in the border zone following myocardial IR. Previous studies have demonstrated that Notch expression is elevated in hearts subjected to myocardial IR injury $(10,11)$. To further determine the area in the infarcted heart in which Notch1 expression is the highest, the levels of Notch1 protein within the infarct, remote and border zones were measured 7 days following IR (CON-IR-7d). Significantly higher expression of Notch1 was observed in border zone compared with the infarct or remote zone (Fig. 6), which suggested that Notch1 may exhibit protective effects in the border zone of infarcted heart, during myocardial IR.

Notch1 expression levels are reduced in the border zone of tail-suspended rat hearts following myocardial IR injury. Notch1 protein expression levels were higher in the border zone compared with expression levels in CON-Sham rats, SUS-Sham rats, and in the infarcted area and the remote zone, of the same age in both CON-IR-7d and SUS-IR-7d rats (Fig. 7). However, Notch1 expression was significantly lower in SUS-IR-7d rats compared with expression levels in CON-IR-7d rats (Fig. 7B).

Infarcted zone size is reduced following myocardial IR. The myocardial infarct size was significantly smaller in rats that underwent 7 days of reperfusion compared to those who underwent $3 \mathrm{~h}$ of reperfusion in both CON and SUS groups (Fig. 8). In addition, the myocardial infarct size was significantly larger in both SUS groups compared with both of the corresponding CON groups post-IR.

\section{Discussion}

The gravitational field is crucial for regulating cardiovascular function. It has been reported that astronauts exhibit decreased cardiac preload, circulating blood volume and red cell mass following long-term exposure to microgravity, which in turn may 

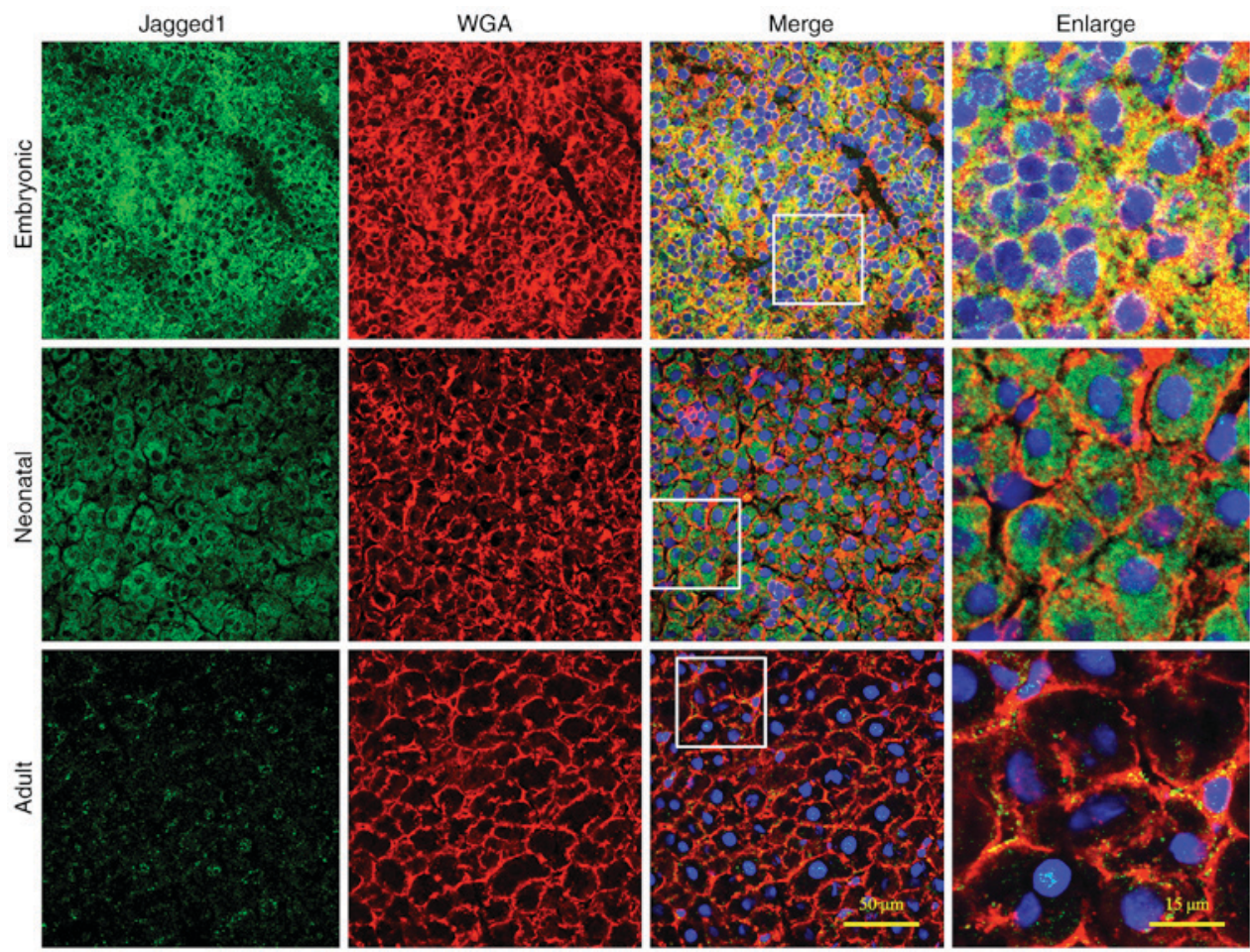

Figure 3. Expression of hepatic JAG1 in different stages of rat development. Frozen sections (magnification, x60) of liver from embryonic, neonatal and adult rats were stained for JAG1 (green), membrane stain (WGA, red) and nucleus (Hoechst, blue). The intensity of JAG1 expression decreased, similar to Notch1 expression. The enlarged area (magnification, x200) indicates the ring-shaped distribution of JAG1 around hepatocytes. JAG1, Jagged1.

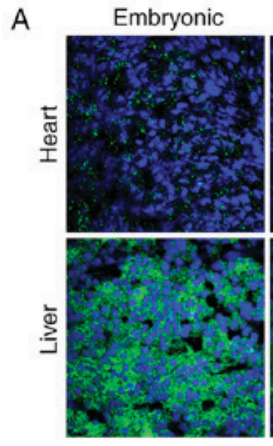

Neonatal

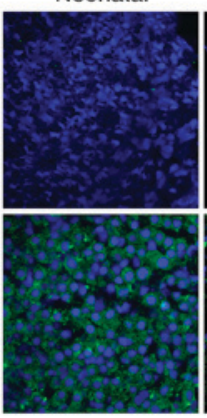

B

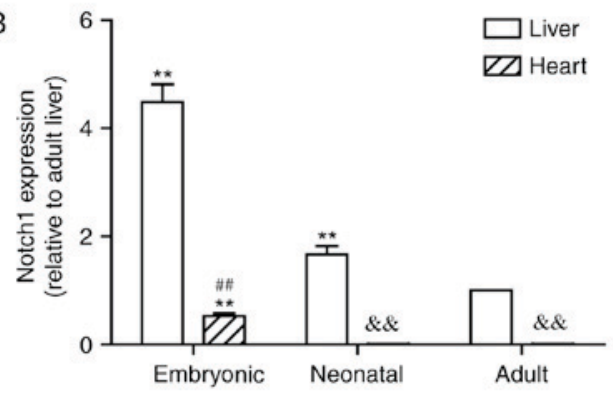

Figure 4. Notch1 expression in rat heart and liver at different stages (A) Frozen sections of heart and liver from embryonic, neonatal and adult rats were stained for the extracellular domain of Notch1 (green) and nucleus (Hoechst, blue). As the tissues developed naturally from the embryonic stage towards the adult stage, the constituent cells of adult groups grew larger than the other groups. Therefore, the tissues of adult rats achieved a lower density than those of embryonic and neonatal rats did. All displayed sections were captured under the same magnification (x60). (B) Quantification of the relative fluorescence intensity of Notch1 expression. The values are shown as fold change vs. the expression levels of Notch1 in adult liver group. Data are presented as the mean \pm standard error of the mean of three experiments. ${ }^{* *} \mathrm{P}<0.01$ vs. the adult liver group; ${ }^{\# \#} \mathrm{P}<0.01$ vs. the embryonic liver group; ${ }^{\&} \mathrm{P}<0.01$ vs. the embryonic heart group.
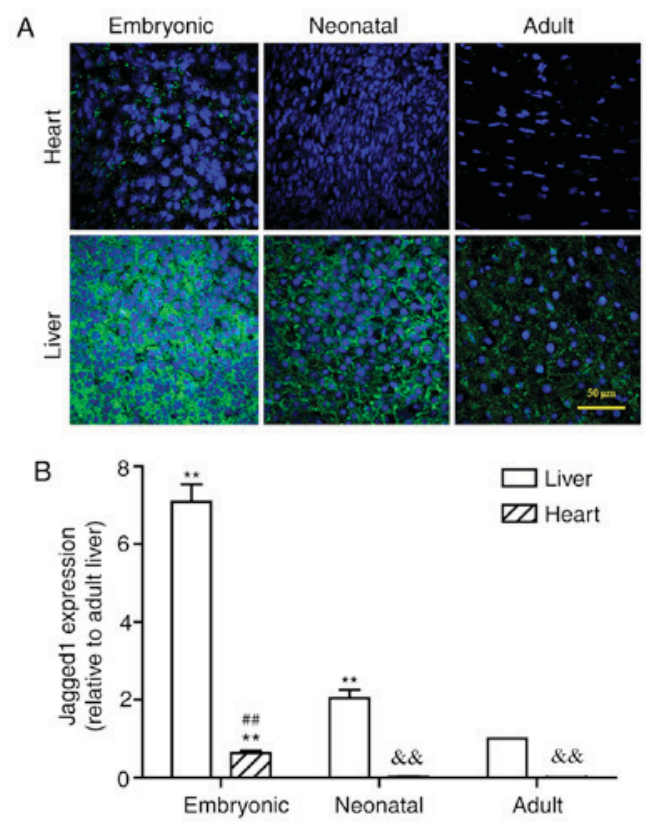

Figure 5. Jagged1 expression in rat heart and liver. (A) Frozen sections of heart and liver from embryonic, neonatal, and adult rats were stained for Jagged1 (green) and nucleus (Hoechst, blue). All displayed sections were captured under the same magnification (x60). (B) Quantification of the relative fluorescence intensity of Jagged 1 expression. The values are shown as fold change vs. the expression levels of Jagged1 in adult liver group. Data are presented as the mean \pm standard error of the mean of three experiments. ${ }^{* * *} \mathrm{P}<0.01$ vs. the adult liver group; ${ }^{\# \#} \mathrm{P}<0.01$ vs. the embryonic liver group; ${ }^{\&} \mathrm{P}<0.01$ vs. the embryonic heart group.

jeopardize the ability of the body to fight against cardiovascular injury (23). Our previous data have demonstrated that the 

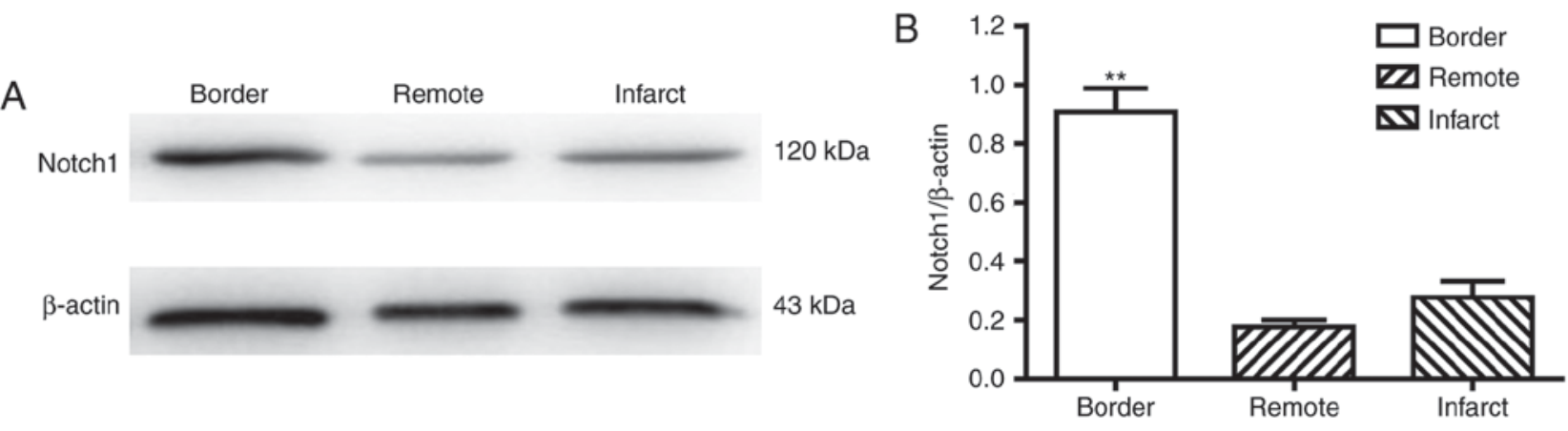

Figure 6. Notch1 protein expression is increased in the border zone 7 days following ischemia-reperfusion injury. (A) Representative western blot images from CON-IR-7d rats indicating the expression levels of Notch1 in the three zones of the heart; $\beta$-actin was used as a loading control. (B) Quantification of Notch1 expression levels from Part (A) normalized to $\beta$-actin expression. Data are presented as the mean \pm standard error of the mean of three experiments. ${ }^{* *} \mathrm{P}<0.01$ vs. remote zone.
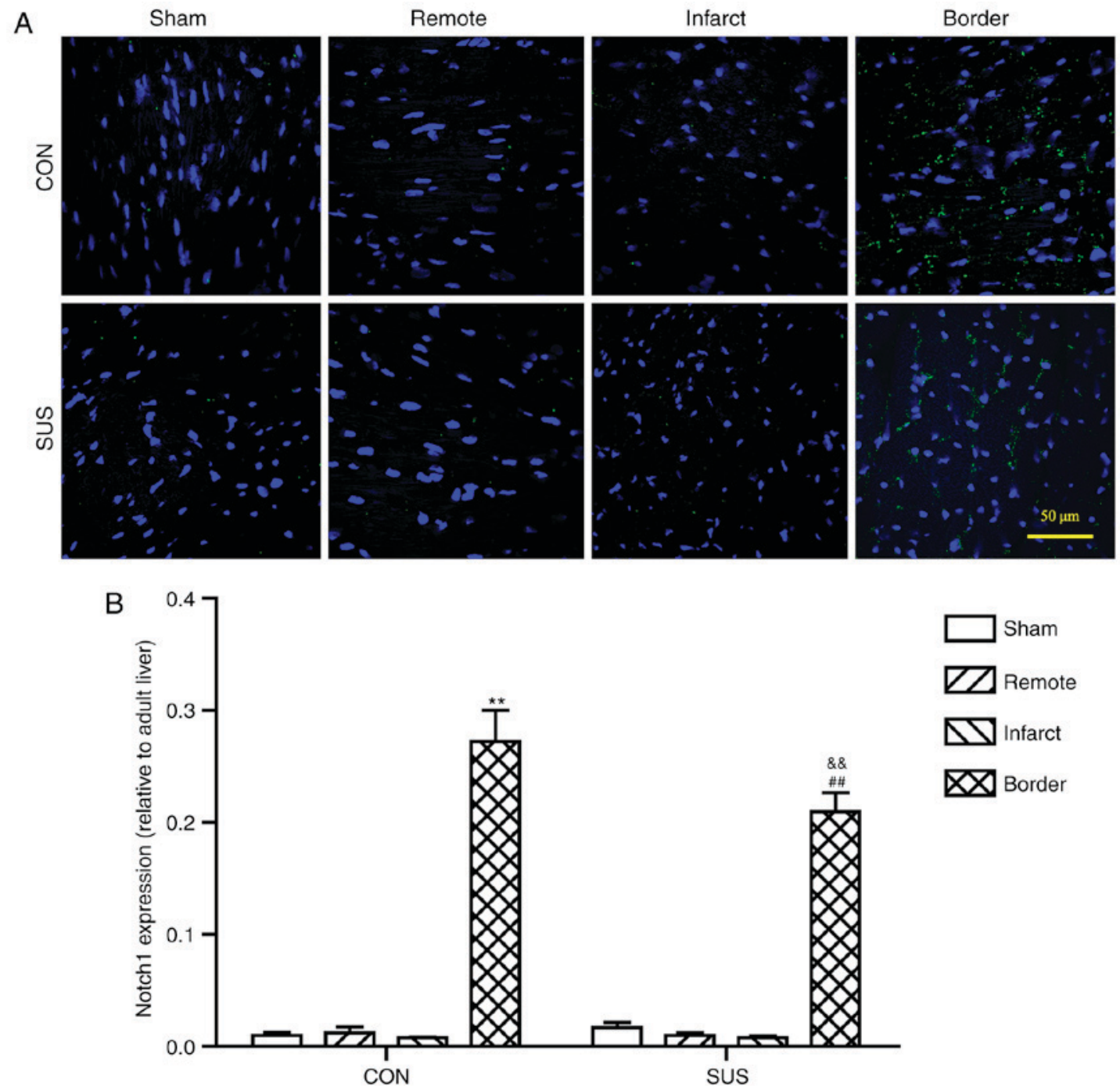

Figure 7. Notch1 expression is increased in the border zone in rat hearts following IR injury. (A) Frozen sections of adult rat hearts subjected to IR, with or without suspension, or sham operation were stained for the extracellular domain of Notch1 (green) and nucleus (Hoechst, blue). All sections were also counterstained with Cx43 (data not shown) to distinguish the different zones of the infarcted hearts. (B) Quantification of the relative fluorescence intensity of Notch1 expression. The values are shown as fold changes vs. the expression levels of Notch1 in adult liver group indicated in Fig. 4. Data are presented as the mean \pm standard error of the mean of three experiments. ${ }^{* *} \mathrm{P}<0.01$ vs. CON-Sham rats; ${ }^{\# /} \mathrm{P}<0.01$ vs. SUS-Sham rats; ${ }^{\text {\&\&}} \mathrm{P}<0.01$ vs. the border zone of $\mathrm{CON}$ rats. CON, control; Cx43, connexin 43; IR, ischemia reperfusion; SUS, tail-suspended.

myocardium is more sensitive to IR injury under microgravity exposure (4). In the present study, a 4-week tail suspension rat model was used to simulate microgravity and to further investigate the detailed mechanisms behind this change. 
A
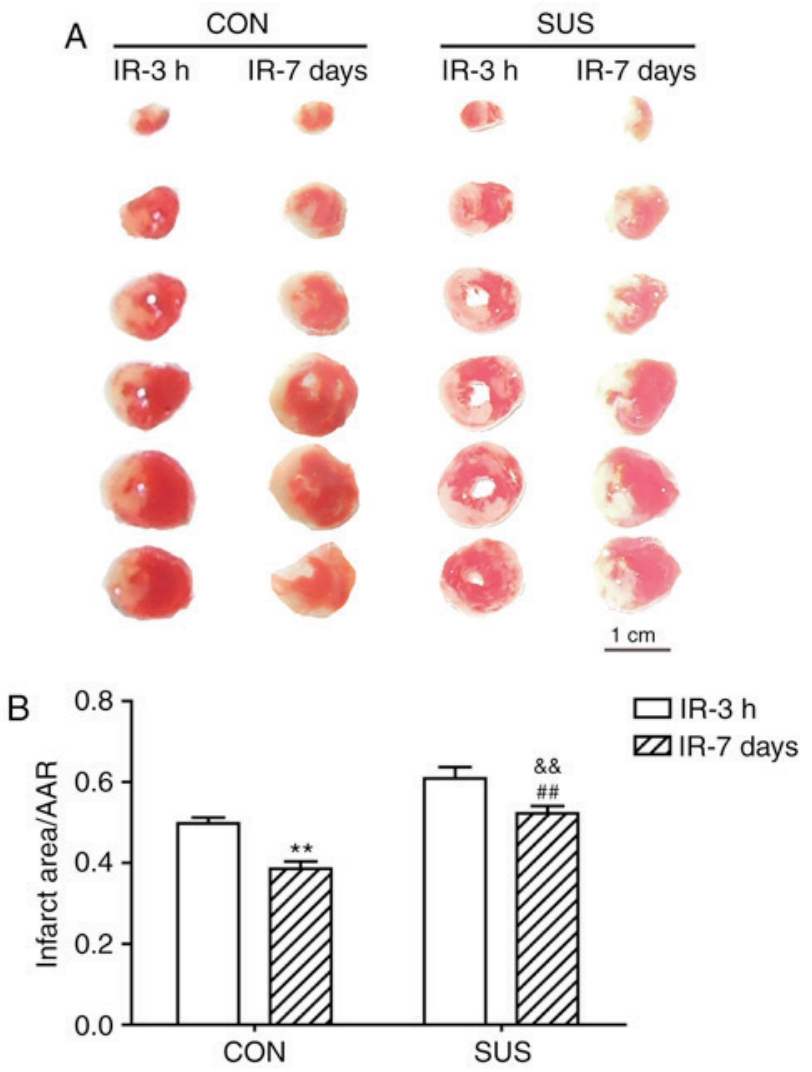

Figure 8. Infarct size of left ventricular myocardium following IR. (A) Representative sections at $3 \mathrm{~h}$ (IR-3h) or 7 days (IR-7d) post-IR. (B) The infarct area was quantified and expressed as percentage of the mass of the left ventricle. Viable tissue is stained red, and infarcted area is white. Data are presented as the mean \pm standard error of the mean of three experiments. ${ }^{* *} \mathrm{P}<0.01$ vs. the IR-3h group of CON rats; ${ }^{* \#} \mathrm{P}<0.01$ vs. the IR-3h group of SUS rats; ${ }^{\& \&} \mathrm{P}<0.01$ vs. the IR-7d group of $\mathrm{CON}$ rats. CON, control; IR, ischemia reperfusion; SUS, tail-suspended; AAR, area at risk.

Notch signaling pathway is a conserved cell-cell interaction system that controls tissue formation and homeostasis during embryonic and adult life (6). Previous studies have demonstrated that Notch serves multifaceted roles in cardiac development, including ventricular chamber development (24) and embryonic vasculature remodeling (25). Additionally, Notch signaling participates in the maintenance of adult heart tissue integrity $(10,26)$. Notch1 is also involved in heart diseases, including myocardial infarction (27) and hypertrophic cardiomyopathy (10). In particular, Notch activation mediates cardioprotection provided by ischemic preconditioning and ischemic postconditioning (28). Previous studies have reported that Notch1 signaling also contributes to the suppression of IR-injury-induced cardiac oxidative/nitrative stress $(12,13)$. Therefore, Notch1 upregulation may represent a potential pharmacologic target for the cardioprotection of ischemic heart disease. However, it remains unknown whether Notch1 functions properly in myocardial IR under microgravity exposure. In the present study, the expression and distribution of Notch1 expression was investigated. Subsequently, the differences in Notch signaling expression following myocardial IR were compared between control and tail-suspended rats following IR injury.

Previous studies have demonstrated the expression of Notch1 and JAG1 in fetal and postnatal rat and human liver tissues $(29,30)$. Since most studies that examined the myocardial Notch1 expression by the NICD levels (12), the present study is the first to the best of the authors' knowledge to confirm that the Notch1 antibody against the extracellular domain can detect myocardial Notch1 levels. Data from the present study also indicated that the hepatic expression of Notch1 and JAG1 declined remarkably from embryo to the adult stage. Importantly, the expression of Notch1 and JAG1 in normal heart is far lower compared with the expression in liver at any developmental stage.

Notch1 has been previously reported to exert cardioprotective effects in myocardial IR injury (13). Owing to the negligible regenerative capacity of the heart, myocardial infarction ultimately leads to the replacement of necrotic cardiomyocytes with a collagen-based scar. A previous study divided the cellular and molecular events involved in the reparative response into three overlapping phases (16). During the inflammatory phase, the activation of cytokines and chemokines induces a protease-rich environment, which in turn leads to extensive degradation of the cardiac matrix (31-33). The transition to the proliferative phase undergoes the inhibition of pro-inflammatory signals and the promotion of fibrous tissue deposition and angiogenesis (34). During the proliferative phase, the restricted expressions of fibronectin and matricellular proteins regulate the phenotype, survival and gene expression of cardiac cells (35). Finally, the formation of a mature cross-linked matrix may 'shield' fibroblasts and further lead to their deactivation during the maturation phase $(36,37)$. Of the three phases, myocardial reparative response mainly occurs during the maturation phase, which in rodents is between 5 and 25 days, and in large mammals is between 14 days and 2 months. Therefore, the present study focused on Notch1 expression during the maturation phase of myocardial IR injury. Notable increases of Notch1 expression were observed in the border zone at 7 days post-IR injury, which was consistent with the study by Gude et al (27).

The border zone surrounding a healed myocardial infarction is a region where the normal organization of cardiac cells gets disrupted (20). Tremendous effort has been devoted to the research on limiting infarct size. Thereinto, the size of the ischemic but still viable border zone has received increasing attention in experimental animals $(38,39)$. The present data indicated a significant increase in Notch1 expression levels at the border zone compared with the remote and the infarcted zones, 7 days post-myocardial IR injury (CON-IR-7d) and may indicate a positive role of Notch signaling in the repair phase of myocardial IR process.

Tail-suspended rat models have been widely used to simulate microgravity to study the influence of weightlessness on the cardiovascular system (17). In the present study, increased Notch1 expression was observed following myocardial IR injury in the border zone compared with the remote zone and infarct zone of tail-suspended rats. However, Notch1 expression levels were reduced in the tail-suspension group compared with the control group. It may be hypothesized that the normal function of Notch1 in protection against myocardial IR injury may be interrupted when exposed to simulated microgravity; however, further in vivo studies are required. 
A link between the function of the infarcted heart and the decreased expression of Notch1 under simulated microgravity was also examined. According to Dobaczewski et al (16), the rat models that underwent $3 \mathrm{~h}$ of reperfusion (IR-3h) were used to represent the situation without repair responses to myocardial IR injury. The IR-3h groups served as the control for comparing the extent of myocardial reparation at $7 \mathrm{~d}$ after reperfusion. The results indicated that the infarcted area was significantly decreased in rats that underwent 7 days of reperfusion compared with those who underwent only $3 \mathrm{~h}$ of reperfusion. Additionally, the infarction area at time points between $3 \mathrm{~h}$ and 7 days, including 6,12 h, 1 and 4 days, and even $1 \mathrm{~h}$ and 14 days (unpublished data) was evaluated. Among them, the damage of the rat heart (infarcted area) peaked at $3 \mathrm{~h}$ following IR injury, when the heart was still in the inflammatory phase. Later, the infarction area gradually decreased and it finally stabilized at $7 \mathrm{~d}$ after reperfusion (Jiang et al, unpublished data). In agreement with the immunohistochemical results, which demonstrated that Notch1 expression was decreased in tail-suspended rats (CON-IR-7d), the myocardial infarct size was significantly increased in tail-suspended rats (SUS-IR-7d) compared with the control rats (CON-IR-7d), which provided further evidence for the presumption that the proper function of Notch signaling pathway was hampered under simulated microgravity.

However, this study has few limitations. First, myocardial Notch signaling pathway mainly involves JAG1, Notch1 and several downstream target genes, including HES1, HEY1 and cyclin D $(40,41)$. In the present study, the main focus was on the distribution of Notch1 expression, and therefore proteins involved in the intracellular action of signaling were not evaluated. Furthermore, the presented data mainly provide morphological evidence that validates the antibody choice and depicts the distribution of Notch. Additional experiments by knockdown or overexpression of Notch1 should be conducted to confirm whether Notch1 signaling is dependent upon simulated microgravity and to what extent simulated microgravity hampers the protective effects of Notch signaling against myocardial IR.

In conclusion, by establishing and validating a tailsuspended rat model with myocardial IR, novel features of Notch1 expression in the repair phase of border zone were observed. The present data confirmed that Notch1 and its endogenous ligand JAG1 are poorly expressed in normal adult heart. In addition, it was demonstrated that there is significantly higher Notch 1 expression in border zone compared to infarct zone and remote zone following myocardial IR. Notably, it was observed for the first time that simulated microgravity may jeopardize the normal function of Notch1 in the fight against myocardial IR. These data may improve our knowledge of the underlying mechanisms of ischemic heart disease in weightless environment, but further studies evaluating the role of Notch1 in myocardial IR injury and the reduction of cardiac ischemic risk in astronauts are needed.

\section{Acknowledgements}

This study was supported by The National Natural Science Foundation of China (grant no. 81571844). We would also like to thank Mr. Cheng-Fei Li (Department of Aerospace Biodynamics, The Fourth Military Medical University, Xi'an, China) for the kind help with Western blot detection.

\section{References}

1. World Health Organization: World Health Statistics 2016-Monitoring health for the SDGs. Sustainable development goals. http://www.who.int/gho/publications/world_health_statistics/2016/EN_WHS2016_TOC.pdf. Accessed April 16, 2016.

2. Ibáñez B, Heusch G, Ovize M and Van de Werf F: Evolving therapies for myocardial ischemia/reperfusion injury. J Am Coll Cardiol 65: 1454-1471, 2015.

3. White RJ and Blomqvist CG: Central venous pressure and cardiac function during spaceflight. J Appl Physiol (1985) 85: 738-746, 1998.

4. Lu YM, Jiao B, Lee J, Zhang L and Yu ZB: Simulated microgravity increases myocardial susceptibility to ischemia-reperfusion injury via a deficiency of AMP-activated protein kinase. Can J Physiol Pharmacol 95: 59-71, 2017.

5. Wharton KA, Johansen KM, Xu T and Artavanis-Tsakonas S: Nucleotide sequence from the neurogenic locus notch implies a gene product that shares homology with proteins containing EGF-like repeats. Cell 43: 567-581, 1985.

6. Niessen K and Karsan A: Notch signaling in cardiac development. Circ Res 102: 1169-1181, 2008.

7. Wiese C, Heisig $\mathrm{J}$ and Gessler M: Hey bHLH factors in cardiovascular development. Pediatr Cardiol 31: 363-370, 2010.

8. Ferrari R and Rizzo P: The Notch pathway: A novel target for myocardial remodelling therapy? Eur Heart J 35: 2140-2145, 2014.

9. Yu HC, Qin HY, He F, Wang L, Fu W, Liu D, Guo FC, Liang L, Dou KF and Han H: Canonical notch pathway protects hepatocytes from ischemia/reperfusion injury in mice by repressing reactive oxygen species production through JAK2/STAT3 signaling. Hepatology 54: 979-988, 2011.

10. Croquelois A, Domenighetti AA, Nemir M, Lepore M, Rosenblatt-Velin N, Radtke F and Pedrazzini T: Control of the adaptive response of the heart to stress via the Notch1 receptor pathway. J Exp Med 205: 3173-3185, 2008.

11. Boccalini G, Sassoli C, Formigli L, Bani D and Nistri S: Relaxin protects cardiac muscle cells from hypoxia/reoxygenation injury: Involvement of the Notch-1 pathway. FASEB J 29: 239-249, 2015.

12. Pei H, Song X, Peng C, Tan Y, Li Y, Li X, Ma S, Wang Q, Huang R, Yang D, et al: TNF- $\alpha$ inhibitor protects against myocardial ischemia/reperfusion injury via Notch1-mediated suppression of oxidative/nitrative stress. Free Radic Biol Med 82: 114-121, 2015.

13. Pei H, Yu Q, Xue Q, Guo Y, Sun L, Hong Z, Han H, Gao E, Qu Y and Tao L: Notch1 cardioprotection in myocardial ischemia/reperfusion involves reduction of oxidative/nitrative stress. Basic Res Cardiol 108: 373, 2013.

14. Greenwald I and Seydoux G: Analysis of gain-of-function mutations of the lin-12 gene of Caenorhabditis elegans. Nature 346: 197-199, 1990.

15. Rebay I, Fleming RJ, Fehon RG, Cherbas L, Cherbas P and Artavanis-Tsakonas S: Specific EGF repeats of Notch mediate interactions with Delta and Serrate: Implications for Notch as a multifunctional receptor. Cell 67: 687-699, 1991.

16. Dobaczewski M, Gonzalez-Quesada C and Frangogiannis NG: The extracellular matrix as a modulator of the inflammatory and reparative response following myocardial infarction. J Mol Cell Cardiol 48: 504-511, 2010.

17. Yu ZB, Zhang LF and Jin JP: A proteolytic NH2-terminal truncation of cardiac troponin I that is up-regulated in simulated microgravity. J Biol Chem 276: 15753-15760, 2001.

18. Gao E, Lei YH, Shang X, Huang ZM, Zuo L, Boucher M, Fan Q, Chuprun JK, Ma XL and Koch WJ: A novel and efficient model of coronary artery ligation and myocardial infarction in the mouse. Circ Res 107: 1445-1453, 2010.

19. Reimer KA, Lowe JE, Rasmussen MM and Jennings RB: The wavefront phenomenon of ischemic cell death. 1. Myocardial infarct size vs. duration of coronary occlusion in dogs. Circulation 56: 786-794, 1977.

20. Rutherford SL, Trew ML, Sands GB, LeGrice IJ and Smaill BH: High-resolution 3-dimensional reconstruction of the infarct border zone: Impact of structural remodeling on electrical activation. Circ Res 111: 301-311, 2012. 
21. Yu ZB, Gao F, Feng HZ and Jin JP: Differential regulation of myofilament protein isoforms underlying the contractility changes in skeletal muscle unloading. Am J Physiol Cell Physiol 292: C1192-C1203, 2007.

22. Maass K, Chase SE, Lin X and Delmar M: Cx43 CT domain influences infarct size and susceptibility to ventricular tachyarrhythmias in acute myocardial infarction. Cardiovasc Res 84: 361-367, 2009

23. Herault S, Fomina G, Alferova I, Kotovskaya A, Poliakov V and Arbeille P: Cardiac, arterial and venous adaptation to weightlessness during 6-month MIR spaceflights with and without thigh cuffs (bracelets). Eur J Appl Physiol 81: 384-390, 2000.

24. Grego-Bessa J, Luna-Zurita L, del Monte G, Bolós V, Melgar P, Arandilla A, Garratt AN, Zang H, Mukouyama YS, Chen H, et al: Notch signaling is essential for ventricular chamber development. Dev Cell 12: 415-429, 2007.

25. Xue Y, Gao X, Lindsell CE, Norton CR, Chang B, Hicks C, Gendron-Maguire M, Rand EB, Weinmaster G and Gridley T: Embryonic lethality and vascular defects in mice lacking the Notch ligand Jagged1. Hum Mol Genet 8: 723-730, 1999.

26. Nemir M and Pedrazzini T: Functional role of Notch signaling in the developing and postnatal heart. J Mol Cell Cardiol 45: 495-504, 2008

27. Gude NA, Emmanuel G, Wu W, Cottage CT, Fischer K, Quijada P, Muraski JA, Alvarez R, Rubio M, Schaefer E and Sussman MA: Activation of Notch-mediated protective signaling in the myocardium. Circ Res 102: 1025-1035, 2008.

28. Zhou XL, Wan L, Xu QR, Zhao Y and Liu JC: Notch signaling activation contributes to cardioprotection provided by ischemic preconditioning and postconditioning. J Transl Med 11: 251, 2013.

29. Köhler C, Bell AW, Bowen WC, Monga SP, Fleig W and Michalopoulos GK: Expression of Notch-1 and its ligand Jagged-1 in rat liver during liver regeneration. Hepatology 39: 1056-1065, 2004.

30. Louis AA, Van Eyken P, Haber BA, Hicks C, Weinmaster G, Taub R and Rand EB: Hepatic jagged1 expression studies. Hepatology 30: 1269-1275, 1999.

31. Cleutjens JP, Kandala JC, Guarda E, Guntaka RV and Weber KT: Regulation of collagen degradation in the rat myocardium after infarction. J Mol Cell Cardiol 27: 1281-1292, 1995.
32. Gaggar A, Jackson PL, Noerager BD, O'Reilly PJ, McQuaid DB, Rowe SM, Clancy JP and Blalock JE: A novel proteolytic cascade generates an extracellular matrix-derived chemoattractant in chronic neutrophilic inflammation. J Immunol 180: 5662-5669, 2008.

33. Spinale FG: Myocardial matrix remodeling and the matrix metalloproteinases: Influence on cardiac form and function. Physiol Rev 87: 1285-1342, 2007.

34. Sottile $\mathbf{J}$ and Hocking DC: Fibronectin polymerization regulates the composition and stability of extracellular matrix fibrils and cell-matrix adhesions. Mol Biol Cell 13: 3546-3559, 2002.

35. Shimazaki M, Nakamura K, Kii I, Kashima T, Amizuka N, Li M, Saito M, Fukuda K, Nishiyama T, Kitajima S, et al: Periostin is essential for cardiac healing after acute myocardial infarction. J Exp Med 205: 295-303, 2008.

36. Hinz B: Formation and function of the myofibroblast during tissue repair. J Invest Dermatol 127: 526-537, 2007.

37. Tomasek JJ, Gabbiani G, Hinz B, Chaponnier C and Brown RA: Myofibroblasts and mechano-regulation of connective tissue remodelling. Nat Rev Mol Cell Biol 3: 349-363, 2002.

38. Albrecht-Schgoer K, Schgoer W, Holfeld J, Theurl M, Wiedemann D, Steger C, Gupta R, Semsroth S, Fischer-Colbrie R, Beer AG, et al: The angiogenic factor secretoneurin induces coronary angiogenesis in a model of myocardial infarction by stimulation of vascular endothelial growth factor signaling in endothelial cells. Circulation 126: 2491-2501, 2012.

39. MacArthur JW Jr, Purcell BP, Shudo Y, Cohen JE, Fairman A, Trubelja A, Patel J, Hsiao P, Yang E, Lloyd K, et al: Sustained release of engineered stromal cell-derived factor 1-alpha from injectable hydrogels effectively recruits endothelial progenitor cells and preserves ventricular function after myocardial infarction. Circulation 128 (11 Suppl 1): S79-S86, 2013.

40. Bray SJ: Notch signalling: A simple pathway becomes complex. Nat Rev Mol Cell Biol 7: 678-689, 2006.

41. Miele L: Notch signaling. Clin Cancer Res 12: 1074-1079, 2006.

(i) This work is licensed under a Creative Commons Attribution 4.0 International (CC BY 4.0) License. 